\title{
A New Deep HST/ACS CMD of I Zw 18: Evidence for Red Giant Branch Stars
}

\author{
A. Aloisi $i^{1,2}$, F. Annibali ${ }^{1}$, J. Mack ${ }^{1}$, M. Tosi ${ }^{3}$, R. van der Marel ${ }^{1}$, \\ G. Clementini ${ }^{3}$, R. A. Contreras ${ }^{3}$, G. Fiorentino ${ }^{3}$, M. Marconi ${ }^{4}$, \\ I. Musella ${ }^{4}$ and A. Saha ${ }^{5}$ \\ ${ }^{1}$ Space Telescope Science Institute, Baltimore, USA \\ email: aloisi@stsci.edu \\ ${ }^{2}$ On Assignment from the Space Telescope Division of the European Space Agency \\ ${ }^{3}$ INAF - Osservatorio Astronomico di Bologna, Bologna, Italy \\ ${ }^{4}$ INAF - Osservatorio Astronomico di Capodimonte, Napoli, Italy \\ ${ }^{5}$ National Optical Astronomy Observatories, Tucson, USA
}

\begin{abstract}
We present results from new deep HST/ACS photometry of I Zw 18, the most metalpoor blue compact dwarf galaxy in the nearby universe. It has been previously argued that this is a very young system that started forming stars only $\lesssim 500$ Gyr ago, but other work has hinted that older ( $\gtrsim 1$ Gyr) red giant branch (RGB) stars may exist in this galaxy. Our deeper data indeed reveal evidence for an RGB. Underlying old ( $\gtrsim 1 \mathrm{Gyr}$ ) populations are therefore present in even the most metal-poor systems, implying that star formation started at $z \gtrsim 0.1$. The RGB tip (TRGB) magnitude and the properties of Cepheid variables identified from our program indicate that $\mathrm{I} \mathrm{Zw} 18$ is farther away $(D=19.0 \pm 1.8 \mathrm{Mpc})$ than previously believed.
\end{abstract}

Keywords. galaxies: dwarf, irregular, starburst, stellar content, distances and redshifts, Cepheids

\section{Introduction}

Within the framework of hierarchical formation, dwarf $\left(M \lesssim 10^{9} \mathrm{M}_{\odot}\right)$ galaxies are the first systems to collapse and start forming stars, supplying the building blocks for the formation of more massive galaxies through merging and accretion. As remnants of this process, present-day dwarfs may have been sites of the earliest star-formation (SF) activity in the universe. However, the most metal-poor $(12+\log (O / H) \lesssim 7.6$, corresponding to $Z \lesssim 1 / 20 \mathrm{Z}_{\odot}$ ) dwarf irregular (dIrr) and blue compact dwarf (BCD) galaxies have been repeatedly pointed out as good candidate "primeval" galaxies in the nearby universe, with possible ages less than 100-500 Myr (e.g., Izotov \& Thuan 1999). If some of these objects turn out to be young galaxies, their existence would support the view that SF in low-mass systems has been inhibited until the present epoch (e.g., Babul \& Rees 1992). The discovery of a nearby primordial galaxy would thus have revolutionary cosmological implications. On the other hand, the lack of such systems would provide strong constraints on the chemical and physical evolution of low-metallicity dIrr/BCDs.

The only direct way to unambiguously infer the evolutionary status of a metal-poor dIrr/BCD galaxy is to resolve it into stars with deep HST observations, and study stellar features in the color-magnitude diagram (CMD). The brightest of these features that contains stars of significant age is the Red Giant Branch (RGB), formed by low-mass stars with ages $\sim 1-13$ Gyr that are burning $H$ in a shell around a He core. In the last 15 years all metal-poor dIrr galaxies in the Local Group and BCDs with $D \lesssim 15 \mathrm{Mpc}$ have been imaged with HST. Interestingly, an RGB has been detected in all those galaxies for which 
photometric data exist that go deep enough to reach the RGB tip (TRGB, brightest phase of the RGB) in the CMD (e.g., SBS 1415+437, Aloisi et al. 2005, and references therein).

The only possible exception so far is the BCD I Zw 18. With an oxygen abundance $12+\log (O / H)=7.2$, corresponding to $Z=1 / 50 \mathrm{Z}_{\odot}, \mathrm{I} \mathrm{Zw} 18$ is the "prototype" member of its class, and the most metal-poor galaxy in the nearby universe. Many HST studies have focused on the evolutionary status of I Zw 18. After other groups had already resolved the brightest individual stars in the galaxy, our group was the first to go deep enough to detect asymptotic giant branch (AGB) stars in HST/WFPC2 images with ages of at least several hundreds Myr and possibly up to a few Gyrs (Aloisi et al. 1999). These results were confirmed by Östlin (2000) through deep HST/NICMOS imaging. More recently, Izotov \& Thuan (2004) presented new deep HST/ACS observations. Their $I$ vs. $V-I \mathrm{CMD}$ shows no sign of an RGB, from which they concluded that the most evolved (AGB) stars are not older than $500 \mathrm{Myr}$ and that I Zw 18 is a bona fide young galaxy. This result was subsequently challenged by Momany et al. (2005) and our group (Tosi et al. 2006) based on a better photometric analysis of the same data. This showed that many red sources do exist at the expected position of an RGB, and that their density in the CMD drops exactly where a TRGB would be expected. However, the small number statistics, the large photometric errors, and the incompleteness at the TRGB, did not allow a conclusive statement about the possible existence of an RGB in I Zw 18 .

\section{New HST/ACS Observations}

We were awarded 24 additional orbits with ACS over a three-month period starting in October 2005 (GO program 10586, PI Aloisi) to better understand the evolutionary status of $\mathrm{I} \mathrm{Zw}$ 18. The observations were obtained in 12 different epochs in F606W and F814W to: (1) build a deeper CMD to search for RGB stars; (2) detect and characterize Cepheids at the lowest metallicity available in the local universe; and (3) use both the Cepheids and a possible TRGB detection to determine an accurate distance to I Zw 18 . In this paper we will present the preliminary results from the new deep photometry. Preliminary results from the study of the variable stars in this metal-poor galaxy are discussed in Fiorentino et al. (2007, in this volume). Detailed descriptions of the observational program, the implications for the evolutionary state and distance of I $\mathrm{Zw} 18$, and the results on Cepheid variability will be presented in forthcoming papers in the refereed literature.

PSF-fitting photometry was performed on deep images that were obtained by combining the exposures in each filter with MultiDrizzle. After application of CTE and aperture corrections, the count rates were transformed to Johnson-Cousins $V$ and $I$ magnitudes. Values shown and discussed hereafter are corrected for $E(B-V)=0.032$ mag of Galactic foreground extinction, but not for any extinction intrinsic to I Zw 18. The archival ACS data in F555W and F814W (GO program 9400, PI Thuan) were also re-processed in a similar manner. The two ACS datasets were then combined using several different approaches and rejection schemes. The results discussed here were obtained by demanding that stars should be detected in all the four deep images ( $V$ and $I$ for both datasets). At the expense of some depth, this approach has the advantage of minimizing the number of false detections and therefore providing relatively "clean" CMDs.

\section{Results and Interpretation}

Figure 1a shows the resulting $I$ vs. $V-I$ CMD of I Zw 18. The CMD shows faint red stars exactly at the position where an RGB would be expected (see the Padua isochrones overplotted in the figure). Figure 2 shows the luminosity function (LF) of the red stars. 

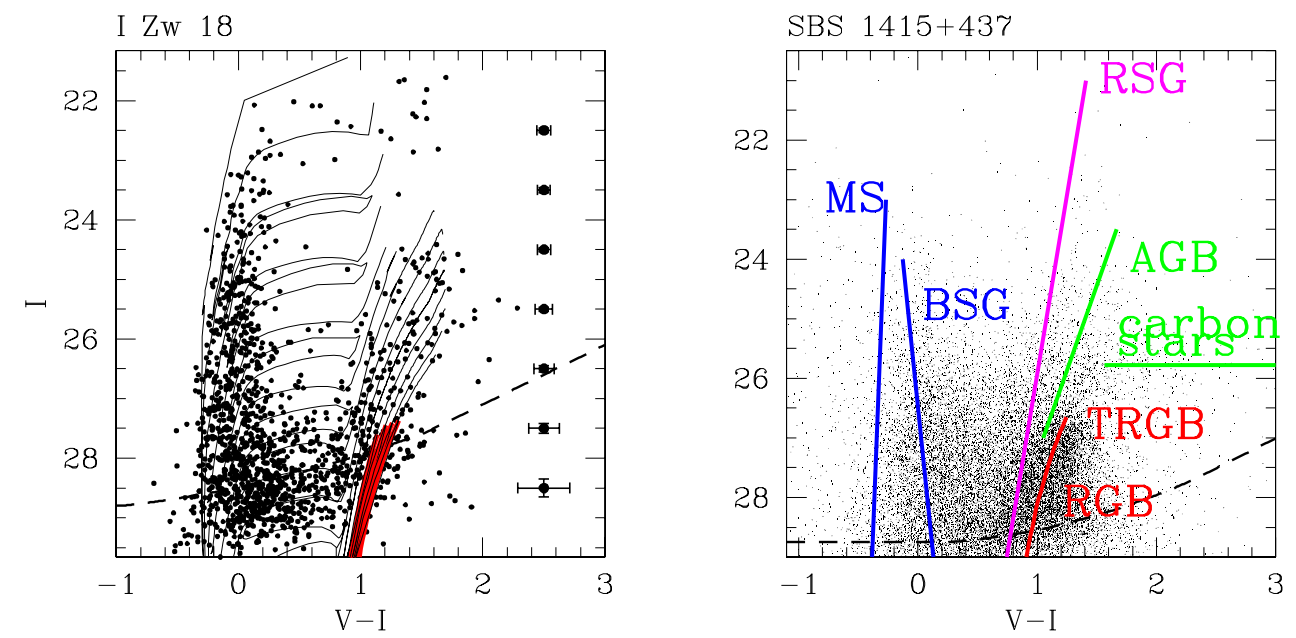

Figure 1. (a) HST/ACS CMD for I Zw 18. Median photometric errors at $V-I=1$ (determined by comparison of measurements from GO-9400 and GO-10586) are shown as function of $I$-band magnitude on the right side of the panel. Padua isochrones from 5.5 Myr to $10 \mathrm{Gyr}$ are overlaid, with the RGB phase for isochrones from 1.7 to 10 Gyr colored red. The isochrones have metallicity $Z=0.0004$ (as inferred from the HII regions of I $\mathrm{Zw} 18$ ) and are shown for the distance $D=19 \mathrm{Mpc}(m-M=31.39)$. The CMD shown here includes stars in both the main and secondary bodies of I Zw 18. (b) HST/ACS CMD for SBS 1415+437 (Aloisi et al. 2005). The main evolutionary sequences seen in the data are indicated in approximate sense as colored straight lines: main sequence (MS), blue supergiants (BSG), red supergiants (RSG), the red giant branch (RGB) with its tip (TRGB), the asymptotic giant branch (AGB), and carbon stars. Both CMDs are corrected for Galactic foreground extinction. Dashed lines are estimates of the $50 \%$ completeness level. The vertical axes of the panels are offset from each other by 0.66 mag, which is the difference in distance modulus between the galaxies (see Fig. 2). Some $\sim 10$ times more stars were detected in SBS $1415+437$, owing to its smaller distance; stars for this galaxy are shown with smaller symbols. The faint red stars in both galaxies indicate that these metal-poor BCD galaxies started forming stars $\gtrsim 1$ Gyr ago.

It shows a sharp drop towards brighter magnitudes, exactly as would be expected from a TRGB. The magnitude of the discontinuity, $I=27.32 \pm 0.10$, implies a distance modulus $m-M=31.39 \pm 0.20$ (e.g., Bellazzini et al. 2001), i.e., $D=19.0 \pm 1.8 \mathrm{Mpc}$. This assumes that the evolved RGB stars have negligible intrinsic extinction. The TRGB distance is consistent with the preliminary analysis of the first few Cepheid variables identified by our program. Comparison of their Wesenheit relation to the Wesenheit relation inferred from new theoretical Cepheid pulsation models at the metallicity of I Zw 18 yields $m-M=31.28 \pm 0.26$ (Fiorentino et al. 2007, in this volume). This agreement further supports our interpretation of the LF drop in Figure 2 as a TRGB feature.

The evidence for an RGB in I Zw 18 is further strengthened by comparison to another BCD, SBS $1415+437$, observed by us with a similar HST/ACS set-up (Aloisi et al. 2005). This galaxy is not quite as metal poor as I $\mathrm{Zw} 18(12+\log (O / H)=7.6)$ and is somewhat nearer at $D \approx 13.6 \mathrm{Mpc}$. But taking into account the differences in distance and completeness, the CMDs of these galaxies look very similar. Since SBS $1415+437$ has an unmistakable RGB sequence, this suggests that such an RGB sequence exists in I Zw 18 as well. It has previously been suggested that I Zw 18 has no stars with ages $\gtrsim 500$ Myr. It is therefore interesting to ask the question whether there exist Star Formation Histories (SFHs) which do not have stars older than $\sim 1$ Gyr (and by extension, do not have an RGB), yet which can still fit: (1) the observed number of faint red stars in I Zw 18 


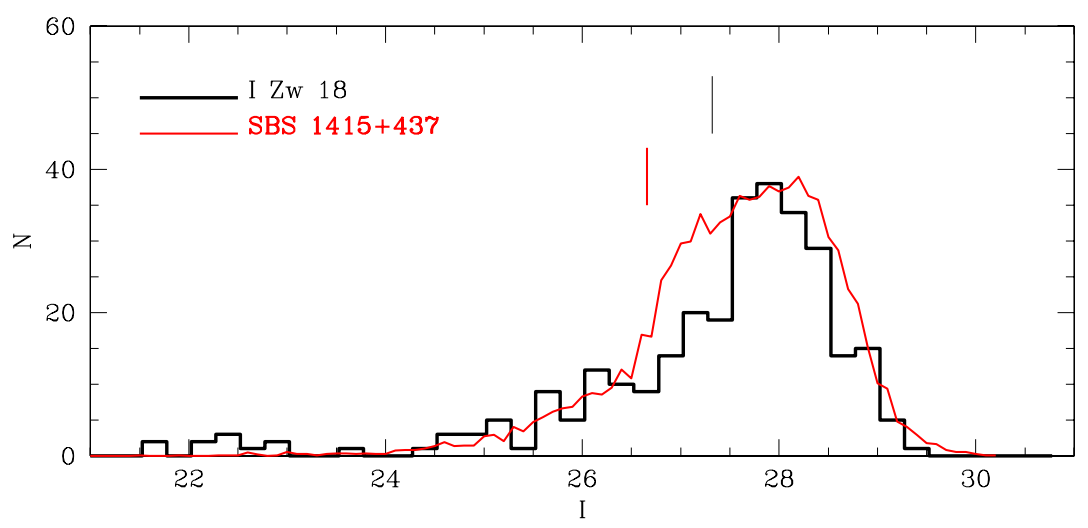

Figure 2. $I$-band LFs for stars with red colors in the range $V-I=0.75-1.5$ mag, inferred from the CMDs in Fig. 1. Normalizations are arbitrary. Vertical marks indicate the positions of the TRGB, as determined using a Savitzky-Golay filtering technique developed by one of us (see Cioni et al. 2000). At these magnitudes there is a steep LF drop towards brighter magnitudes, due to the end of the RGB sequence. By contrast, the LF drop towards fainter magnitudes at $I \gtrsim 28 \mathrm{mag}$ is due to incompleteness in both cases. Apart from a shift $\Delta(m-M) \approx 0.66$, these metal-poor BCD galaxies have very similar LFs.

$(V-I \gtrsim 1.0, I \gtrsim 27.3)$; and $(2)$ the observed LF drop-off at $I \approx 27.3$. Preliminary results of SFH modeling that we have performed indicate that such SFHs do not exist.

\section{Conclusions}

We have obtained new deep HST/ACS observations of I Zw 18 that provide improved insight into the evolutionary status of this benchmark metal-poor BCD. Our results indicate that this galaxy contains RGB stars, in agreement with findings for other local metal-poor BCDs studied with HST. Underlying old ( $\gtrsim 1$ Gyr) populations are therefore present in even the most metal-poor systems, so they must have started forming stars at $z \gtrsim 0.1$. Deeper studies (well below the TRGB) will be needed to pinpoint the exact onset of star formation in these galaxies. We find that at $D=19.0 \pm 1.8 \mathrm{Mpc}$, I Zw 18 is more distant than the values $\sim 15 \mathrm{Mpc}$ that have often been assumed in previous work. This may explain why it has remained difficult for so long to unambiguously detect or rule out the presence of old resolved (RGB) stars in this object. The data that we are compiling on Cepheid stars in I Zw 18 will be unique for probing the properties of variable stars at metallicities that have never before been probed.

\section{Acknowledgements}

Support for proposals \#9361 and \#10586 was provided by NASA through a grant from STScI, which is operated by AURA, Inc., under NASA contract NAS 5-26555.

\section{References}

Aloisi, A., Tosi, M., \& Greggio, L. 1999, AJ 118, 302

Aloisi, A., van der Marel, Mack, J., Leitherer, C., Sirianni, M., \& Tosi, M. 2005, ApJ 631, L45

Babul, A., \& Rees, M. J. 1992, MNRAS 255, 346

Bellazzini, M., Ferraro, F. R., \& Pancino, E. 2001, ApJ, 556, 635

Cioni, M.-R. L., van der Marel, R. P., Loup, C., \& Habing, H. J. 2000, A\&A, 359, 601

Izotov, Y. I., \& Thuan, T. X. 1999, ApJ 511, 639

Izotov, Y. I., \& Thuan, T. X. 2004, ApJ 616, 768 
Momany, Y., et al. 2005, A\&BA 439, 111

Östlin, G. 2000, ApJ 535, L99

Tosi, M., Aloisi, A., Mack, J., \& Maio, M. 2006, Proc. IAU Symp. 235, eds. F. Combes \& J. Palous, in press

\section{Discussion}

GEISLER: What are the implications of your osbservations of very low metallicity Cepheids in IZw18 for the metallicity dependance of the P-L relation?.

ALOISI: From the theoretical models, the Naples group finds that there is no metallicity dependance from the LMC down to IZw18 abundances. In fact, as G. Fiorentino pointed out to me yesterday, also in the empirical relation that corrects for metallicity effects in the Sakkai et al. paper, you can see two regimes, a plateau from very low metallicities up to the LMC metallicity, and then a decline. Sakkai fitted everything with a slope, but it could be that also empirically you have no metallicity dependance up to the LMC.

FErguson: Could you comment on how your metallicity inferred from RGB compares to that in the gas phase, and on the derived chemical evolution from your SFH-fitting?.

Aloisi: We used Padua models at the lowest metallicity of the HII regions in IZw18, so in our model we do not have chemical evolution.

MARAston: The detection of the RGB in IZw18 does not imply that the galaxy is primordial. The RGB can develop at ages as low as 0.6-0.7 Gyr, as observed in the Magellanic Clouds clusters. Can you provide further evidence that the RGB is old (> several Gyrs.?.

Aloisi: A young RGB (i.e., younger than 1 Gyr) would be about 1 magnitude fainter than an older RGB. This owuld imply a larger distance from the TRGB. The fact that our modelling of the SFH with a quite old RGB gives a distance of $\sim 20 \pm 2 \mathrm{Mpc}$, which agrees the the Cepheid distance of $18 \pm 2 \mathrm{Mpc}$ makes us feel confident that the RGB is older than $\sim 1$ Gyr. All this is of course model dependent, and we used Padova 1994 stellar evolution models for our work.

Cid-Fernandes: It is good to see IZw18 question being answered once and for all. In a recent paper on Ultra Compact Blue Dwarf Galaxies we also find a mass dominant old $\sim 10$ Gyr population from fits to the integrated spectra. It would be very interesting to compare the SF histories derived by this method with the more detailed CMD work you have presented here.

ALOISI: I perfectly agree with you. We should do it!. 